\title{
Processamento de licores tipo creme como alternativa para o aproveitamento de resíduos agroindustriais
}

\section{Bruno Fonsêca Feitosa ${ }^{1}, *$ Emanuel Neto Alves de Oliveira ${ }^{2}$, Juvêncio Olegário de Oliveira Neto ${ }^{1}$, Antonio Mateus Tavares Farias $^{3}$ e Regilane Marques Feitosa ${ }^{4}$}

\author{
${ }^{1}$ Universidade Federal de Campina Grande. Campus Pombal. Centro de Ciências e \\ Tecnologia Agroalimentar. Rua Jairo Vieira Feitosa, 1770. Pombal-PB, Brasil \\ (CEP 58840-000).*E-mail: brunofonsecafeitosa@live.com. \\ ${ }^{2}$ Instituto Federal do Rio Grande do Norte. Campus Pau dos Ferros. Rua Antonieta \\ Germano, S/№. Pau dos Ferros-RN, Brasil (CEP 59900-000). \\ ${ }^{3}$ Universidade Federal do Rio Grande do Norte. Campus Natal Central. Rua Lagoa \\ Nova, 1524. Natal-RN, Brasil (CEP 59078-970). \\ ${ }^{4}$ Instituto Federal de Alagoas. Campus Piranhas. Avenida Sergipe, 1521. Piranhas- \\ AL, Brasil (CEP 57460-000).
}

Resumo. A produção de bebidas alcoólicas, como licores, pode ser uma alternativa para o aproveitamento de resíduos da agroindústria processadora de polpa de frutas. Estes resíduos em sua maioria são compostos por nutrientes e podem desempenhar funções fisiológicas importantes. Assim, objetivou-se com esta pesquisa avaliar a viabilidade de processamento de licores tipo creme com resíduos de acerola, abacaxi, manga, goiaba e graviola provenientes da agroindústria de polpa de frutas. Os licores foram elaborados com resíduos secos e triturados de abacaxi, acerola, goiaba, graviola e manga, além de xarope de sacarose e destilado alcoólico. 0 processamento seguiu com maceração alcoólica, filtração, adição de xarope de sacarose, tratamento térmico e acondicionamento. Em seguida, foram avaliadas a qualidade microbiológica, os parâmetros físicos e químicos, o conteúdo de antocianinas e flavonoides, colorimetria, assim como o estudo da cinética de degradação da cor e análise sensorial, quanto a aceitação sensorial, índice de aceitabilidade e intenção de compra. Os resultados obtidos foram tratados utilizando a Análise de Variância, em Delineamento Inteiramente Casualizado, comparando-se as médias pelo teste de Tukey, a $5 \%$ de significância. Os licores tipo creme atenderam aos padrões de identidade e qualidade preconizados pela legislação, com condições higiênico-sanitárias adequadas para o consumo. Pela legislação vigente, os licores elaborados a partir de resíduos agroindustriais nesta pesquisa foram denominados como "tipo creme". A baixa atividade de água associada ao baixo $\mathrm{pH}$, teor alcoólico e concentração de sólidos tende a limitar a multiplicação de microrganismos e favorecer a conservação desses produtos.

Recebido

03/05/2020

Aceito

29/08/2020

Publicado

$31 / 08 / 2020$

Acesso aberto

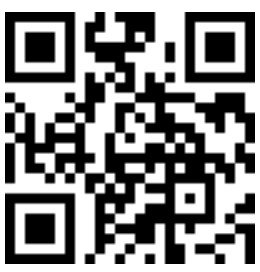

ORCID

(D) 0000-0002-4183-3960 Bruno Fonsêca Feitosa

ISSN 2359-1412/RBGAS-2020-00071/2020/7/16/33/995

Rev. Bras. Gest. Amb. Sustent.

http://revista.ecogestaobrasil.net 
Observou-se um maior conteúdo de antocianinas nos licores de acerola $(4,86 \mathrm{mg} / 100 \mathrm{~g})$ e manga $(5,38 \mathrm{mg} / 100 \mathrm{~g})$. 0 teor de flavonoides foi superior principalmente no licor de acerola, com $40,72 \mathrm{mg} / 100 \mathrm{~g}$. 0 licor de goiaba foi o mais estável durante a cinética de degradação da cor para luminosidade. Os licores apresentaram boa aceitação sensorial, com índice de aceitabilidade acima de $70 \%$. Portanto, o processamento de licores com resíduos agroindustriais pode ser viável como mais uma fonte de renda para os produtores de polpa de frutas.

Palavras-chave: Ananas comosus; Bebidas alcoólicas por mistura; Psidium guajava.

Abstract. Processing of cream liquors as an alternative for the use of agro-industrial waste. The production of alcoholic beverages, such as liqueurs, can be an alternative for the use of residues from the fruit pulp processing agribusiness. These residues are mostly composed of nutrients and can play important physiological functions. Thus, the objective of this research was to evaluate the viability of processing cream-type liqueurs with residues of acerola, pineapple, mango, guava and soursop from the fruit pulp agribusiness. The liqueurs were made with dried and crushed pineapple, acerola, guava, soursop and mango residues, in addition to sucrose syrup and alcoholic distillate. Processing continued with alcoholic maceration, filtration, addition of sucrose syrup, heat treatment and conditioning. Then, microbiological quality, physical-chemical parameters, anthocyanins and flavonoids content, colorimetry were evaluated, as well as the study of color degradation kinetics and sensory analysis, regarding sensory acceptance, acceptability index and purchase intention. The results obtained were treated using the analysis of variance, in a completely randomized design, comparing the averages by the Tukey test, at $5 \%$ significance. Cream-type liqueurs met the standards of identity and quality recommended by law, with hygienic-sanitary conditions suitable for consumption. According to current legislation, liqueurs made from agro-industrial residues in this research were called "cream type". The low water activity associated with low pH, alcohol content and solids concentration tends to limit the multiplication of microorganisms and favor the conservation of these products. A higher anthocyanin content was observed in the acerola $(4.86 \mathrm{mg} / 100 \mathrm{~g})$ and mango $(5.38 \mathrm{mg} / 100 \mathrm{~g})$ liquors. The flavonoid content was higher mainly in the acerola liquor, with $40.72 \mathrm{mg} / 100 \mathrm{~g}$. Guava liquor was the most stable during the color degradation kinetics for luminosity. The liquors showed good sensory acceptance, with an acceptability index above $70 \%$. Therefore, the processing of liquors with agro-industrial residues can be viable as another source of income for fruit pulp producers.

Keywords: Ananas comosus; Alcoholic drinks by mixing; Psidium guajava.
(D) 0000-0002-7000-8126

Emanuel Neto Alves de Oliveira

(1) 0000-0003-2765-5835

Juvêncio Olegário de

Oliveira Neto

(1) $0000-0001-9457-4109$

Antonio Mateus

Tavares Farias

(1) 0000-0003-2627-0933

Regilane Marques

Feitosa 


\section{Introdução}

No Brasil, a fruticultura possui destaque pela promoção da economia, através da geração de renda para agricultores de origem familiar e desenvolvimento do agronegócio (Coelho et al., 2014). Em 2019, as exportações de frutas atingiram 980 milhões de toneladas, com aumento de 16\% em relação a 2018 (ABRAFRUTAS, 2019).

O Nordeste brasileiro dispõe de condições climáticas propícias para a produção de frutas tropicais, que possuem valor econômico, social e alimentar. Com a produção de polpas de frutas congeladas é possível dispor de produtos naturais para a fabricação alimentícia por um maior tempo de estocagem (Oliveira et al., 2018).

A agroindústria processadora de polpa de frutas gera significativa quantidade de resíduos agroindustriais (Feitosa et al., 2019). Nos últimos anos aumentou o interesse científico com o aproveitamento de resíduos, como cascas, sementes, talos, entre outras partes comumente descartadas (Uchôa Thomaz et al., 2014; Oliveira et al., 2019).

Os resíduos agroindustriais são compostos por nutrientes, como vitaminas, minerais e fibras, além de antioxidantes com importância para as funções fisiológicas (Infante et al., 2013). 0 processamento de novos produtos de panificação, doces e bebidas é uma alternativa para o aproveitamento de resíduos (Oliveira et al., 2018).

As bebidas alcoólicas ocupam um lugar de destaque nas mais diversas civilizações já existentes (Santos et al., 2018). A venda brasileira anual de licores atingiu cerca de 7 milhões de litros, evoluindo quanto as tecnologias empregadas e diversificação dos sabores produzidos (SEBRAE, 2014).

A legislação brasileira define licor como uma bebida alcoólica por mistura que possui graduação alcoólica entre 15 e $54 \%$ v/v e um percentual de açúcar superior 30 g.L1. É elaborado com álcool de cereais potável de origem agrícola ou destilado alcoólico, sendo adicionado de extratos ou substâncias aromatizantes, saborizantes, corantes ou outros aditivos permitidos (Brasil, 2008; 2009).

Assim, objetivou-se com esta pesquisa avaliar a viabilidade de processamento de licores tipo creme com resíduos de acerola, abacaxi, manga, goiaba e graviola provenientes da agroindústria de polpa de frutas.

\section{Materiais e métodos}

Os licores foram elaborados no Laboratório de Processamento de Alimentos do Instituto Federal de Educação, Ciência e Tecnologia do Rio Grande do Norte (IFRN), campus Pau dos Ferros. Foram utilizados cinco tipos distintos de resíduos agroindustriais oriundos da produção de polpas de frutas, fornecidos pela empresa Nossa Fruta ${ }^{\circledR}$, localizada no Município de Pereiro, no Estado do Ceará, nordeste do Brasil.

Os resíduos de abacaxi, acerola, goiaba, graviola e manga foram transportados em caixas térmicas hermeticamente vedados e sob refrigeração $\left(6^{\circ} \mathrm{C} \pm 2{ }^{\circ} \mathrm{C}\right)$, da agroindústria de polpas ao laboratório. Posteriormente, foram secos em estufa de circulação forçada de ar (Tecnal®, TE $349 / 3$ ) a $60^{\circ} \mathrm{C}$, por $50 \mathrm{~h}$.

Após a secagem, os resíduos foram triturados em liquidificador industrial (SKYMSEN囚) e peneirados em peneira de 15 mesh, sendo embalados em sacos plásticos com capacidade para 500 g e selados a vácuo em seladora (R.BAIÃO®, BS320). Para o processamento dos licores, considerou-se testes preliminares e a metodologia adequada/empregada por Silva et al. (2017).

Utilizou-se xarope de sacarose (aquecimento de uma mistura de sacarose e água, na proporção $2: 1$, até $70^{\circ}$ Brix) e destilado alcoólico com graduação de $39 \% \mathrm{v} / \mathrm{v}$. Os ingredientes estão apresentados na Tabela 1. 
Tabela 1. Proporção dos ingredientes e rendimento dos licores.

\begin{tabular}{cccccc}
\hline \multirow{2}{*}{ Formulações } & \multicolumn{5}{c}{ Ingredientes } \\
\cline { 2 - 6 } & Resíduos $(\mathrm{g})$ & $\mathrm{DA}(\mathrm{mL})$ & $\mathrm{RM}(\mathrm{mL})$ & XS $(\mathrm{mL})$ & $\mathrm{V}_{\mathrm{f}}(\mathrm{mL})$ \\
\hline $\mathrm{L}_{1}$ & 200 & 815 & 305 & 152,5 & 457,5 \\
$\mathrm{~L}_{2}$ & 200 & 815 & 415 & 207,5 & 622,5 \\
$\mathrm{~L}_{3}$ & 200 & 450 & 290 & 145,0 & 435,0 \\
$\mathrm{~L}_{4}$ & 200 & 450 & 240 & 120,0 & 360,0 \\
$\mathrm{~L}_{5}$ & 200 & 450 & 200 & 100,0 & 300,0 \\
\hline
\end{tabular}

$\mathrm{L}_{1}$ - licor de abacaxi, $\mathrm{L}_{2}$ - licor de acerola, $\mathrm{L}_{3}$ - licor de goiaba, $\mathrm{L}_{4}$ - licor de graviola, $\mathrm{L}_{5}$ - licor de manga; DA - destilado alcoólico, RM - rendimento do macerado, XS - xarope de sacarose, $\mathrm{V}_{\mathrm{f}}$ volume final.

Os resíduos foram pesados e acondicionados em recipientes de vidro higienizados e bem tampados com o destilado alcoólico, correspondendo a etapa de maceração alcoólica. A mistura foi mantida em repouso a temperatura ambiente média de $27{ }^{\circ} \mathrm{C} \pm 4$ ${ }^{\circ} \mathrm{C}$, durante 20 dias (agitando-se suavemente a cada $24 \mathrm{~h}$.).

Posteriormente, os extratos hidroalcoólicos foram filtrados e adicionados do xarope de sacarose ( $50 \%$ do produto final). Em recipiente de polietileno, as bebidas foram submetidas a tratamento térmico em banho-maria a $60^{\circ} \mathrm{C}$, por $2 \mathrm{~h}$.

0 processamento seguiu o fluxograma descrito na Figura 1, sendo os produtos representados pela Figura 2.

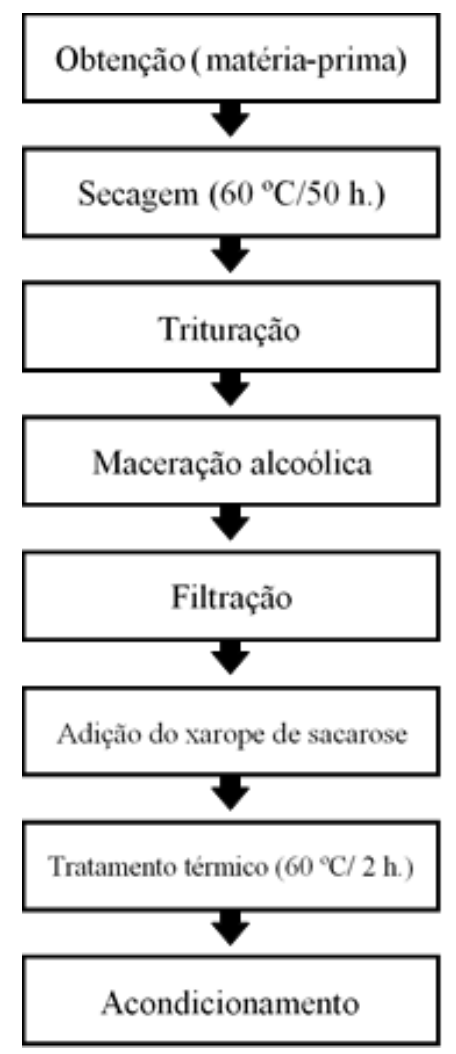

Figura 1. Fluxograma de processamento dos licores. 


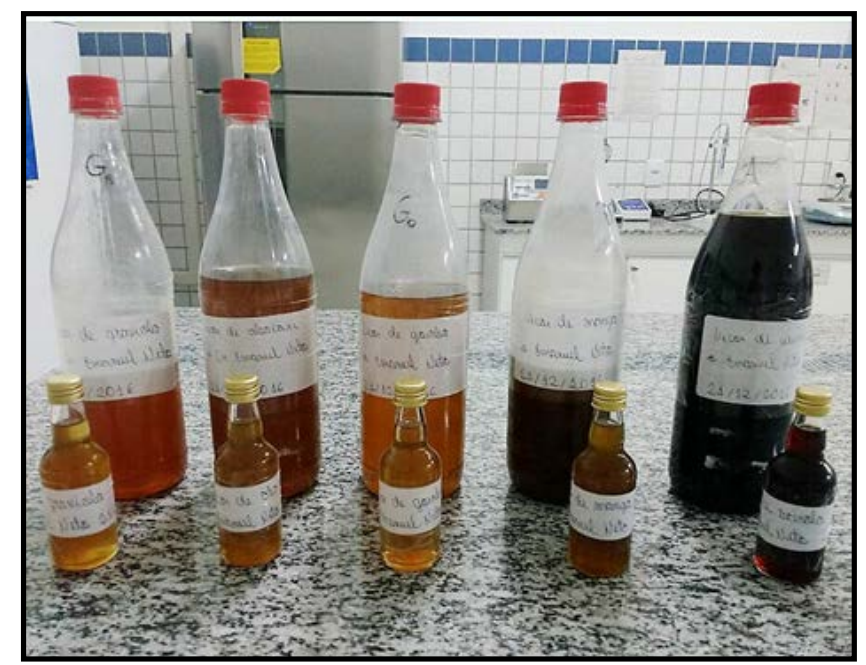

Figura 2. Licores da esquerda para direita: $\mathrm{L}_{4}$ (graviola), $\mathrm{L}_{1}$ (abacaxi), $\mathrm{L}_{3}$ (goiaba), $\mathrm{L}_{5}$ (manga) e $\mathrm{L}_{2}$ (acerola).

A qualidade microbiológica dos licores foi avaliada no Laboratório de Microbiologia de Alimentos do IFRN, campus Pau dos Ferros, em relação à contagem de Coliformes a $35^{\circ} \mathrm{C}$ e Coliformes a $45^{\circ} \mathrm{C}$ (NMP/g), segundo Silva et al. (2017).

Foram avaliados os parâmetros físicos e químicos dos licores, em triplicata, quanto a resíduo mineral, acidez total titulável, pH, açúcares totais e teor alcoólico (IAL, 2008). Avaliou-se ainda a atividade de água em analisador portátil a $30{ }^{\circ} \mathrm{C}$ (Novasina ${ }^{\circledR}$, modelo Labstart) e sólidos solúveis totais, em refratômetro digital (Instrutherm ${ }^{\circledR}$, modelo RT-82).

0 conteúdo de antocianinas e flavonoides foi determinado nos licores pelos métodos descritos por Francis (1982) e Zhishen et al. (1999), respectivamente. A análise colorimétrica foi avaliada, através de leitura direta em espectrofotômetro portátil com sistema de leitura CIELab, iluminante D65 e ângulo de observação de $10^{\circ}$ (MiniScan HunterLab ${ }^{\circledR}$, XE Plus modelo 4500 L), com obtenção dos parâmetros: L* - luminosidade; $a^{*}$ - coloração na região do vermelho $(+a)$ ao verde $(-a)$; e b* - coloração no intervalo do amarelo (+b) ao azul (-b). Com os dados de $a^{*}$ e b* foram calculados: o croma (c*), que corresponde à saturação ou intensidade da cor, sendo $0=$ cor impura e $60=$ cor pura; e 0 ângulo da tonalidade $\left(\mathrm{h}^{*}\right)$, que corresponde a cor perceptível em que $0^{\circ}=$ vermelho, $90^{\circ}=$ amarelo, $180^{\circ}=$ verde, $270^{\circ}=$ azul e $360^{\circ}=$ preto, de acordo com Sensing (1998).

Os licores foram submetidos ao estudo da cinética de degradação da cor (luminosidade, intensidade de vermelho e intensidade de amarelo), avaliados diariamente durante 15 dias, sob temperatura ambiente do Município de Campina Grande-PB e em recipientes herméticos de vidro. Os modelos cinéticos de ordem zero, primeira ordem e segunda ordem (Tabela 2) foram ajustados aos dados experimentais, determinando-se as constantes da velocidade da reação $(k)$.

Assim, o coeficiente de determinação $\left(\mathrm{R}^{2}\right)$ foi utilizado para determinar o melhor modelo matemático. Para calcular os tempos de meia vida $\left({ }^{\Theta_{(1 / 2)}}\right)$ expresso na Tabela 2, utilizou-se o valor de $k$ do modelo cinético que melhor se ajustou aos dados experimentais das cinéticas de degradação da cor. 
Tabela 2. Modelos cinéticos de zero, primeira e segunda ordem e o tempo de meia vida.

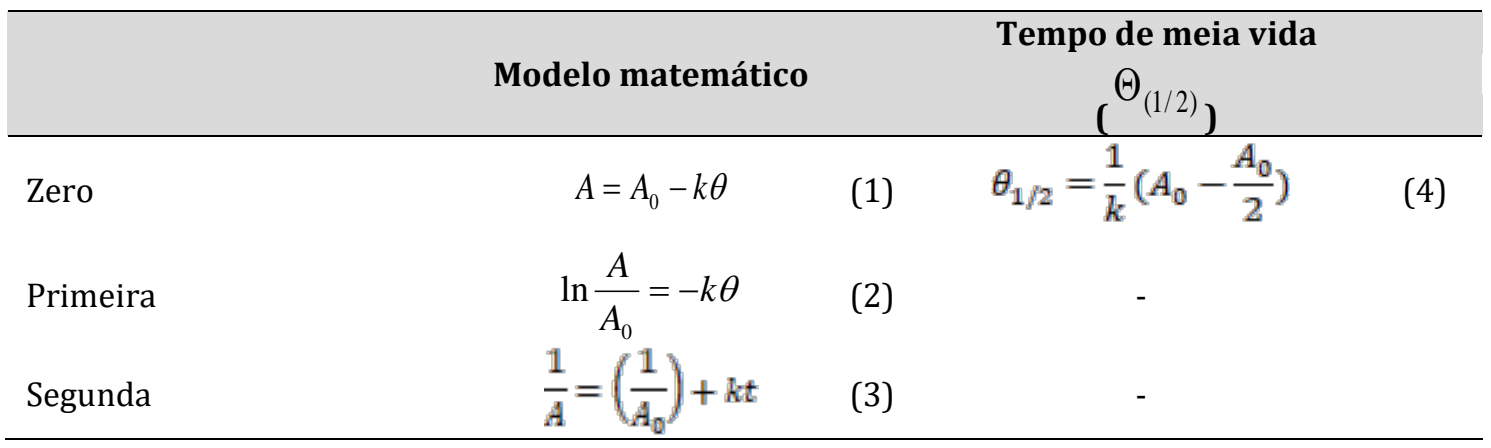

A - concentração do parâmetro avaliado periodicamente; $A_{0}$ - concentração inicial do parâmetro avaliado; k - constante da velocidade da reação; $\theta_{-}$tempo.

A análise sensorial dos licores foi realizada de acordo com a metodologia de Dutcosky (2013), com 60 avaliadores (>18 anos) não treinados. A aceitação sensorial foi avaliada quanto aos atributos cor, aparência, aroma, consistência, sabor, doçura, percepção do teor alcoólico e impressão global, por meio de uma escala hedônica de nove pontos: 9 - gostei muitíssimo, 8 - gostei muito, 7 - gostei moderadamente, 6 - gostei ligeiramente, 5 - nem gostei/nem desgostei, 4 - desgostei ligeiramente, 3 - desgostei moderadamente, 2 - desgostei muito e 1 - desgostei extremamente.

Foi calculado o Índice de Aceitabilidade (IA), conforme a Eq. 5, considerando M como a nota média geral obtida pelo atributo e $\mathrm{N}$ como o número de pontos da escala hedônica (Gularte, 2009).

$I A(\%)=\frac{M}{N} * 100$

A intenção de compra também foi analisada, através da escala hedônica de cinco pontos: 5 - certamente compraria o produto, 4 - possivelmente compraria o produto, 3 talvez comprasse, talvez não comprasse, 2 - possivelmente não compraria o produto e 1 certamente não compraria o produto (Dutcosky, 2013).

Os resultados obtidos na caracterização física e química e aceitação sensorial foram tratados, utilizando a Análise de Variância (ANOVA), em Delineamento Inteiramente Casualizado (DIC). Com o auxílio do software Assistat versão 7.7 beta (Silva e Azevedo, 2016), comparou-se as médias pelo teste de Tukey, a nível de 5\% de significância $(\mathrm{p}<0,05)$.

\section{Resultados e discussão}

Na Figura 3 estão apresentados os valores médios dos parâmetros físicos e químicos de açúcares totais e teor alcoólico e na Figura 4 está expressa a correlação de pH e acidez total titulável dos licores. 


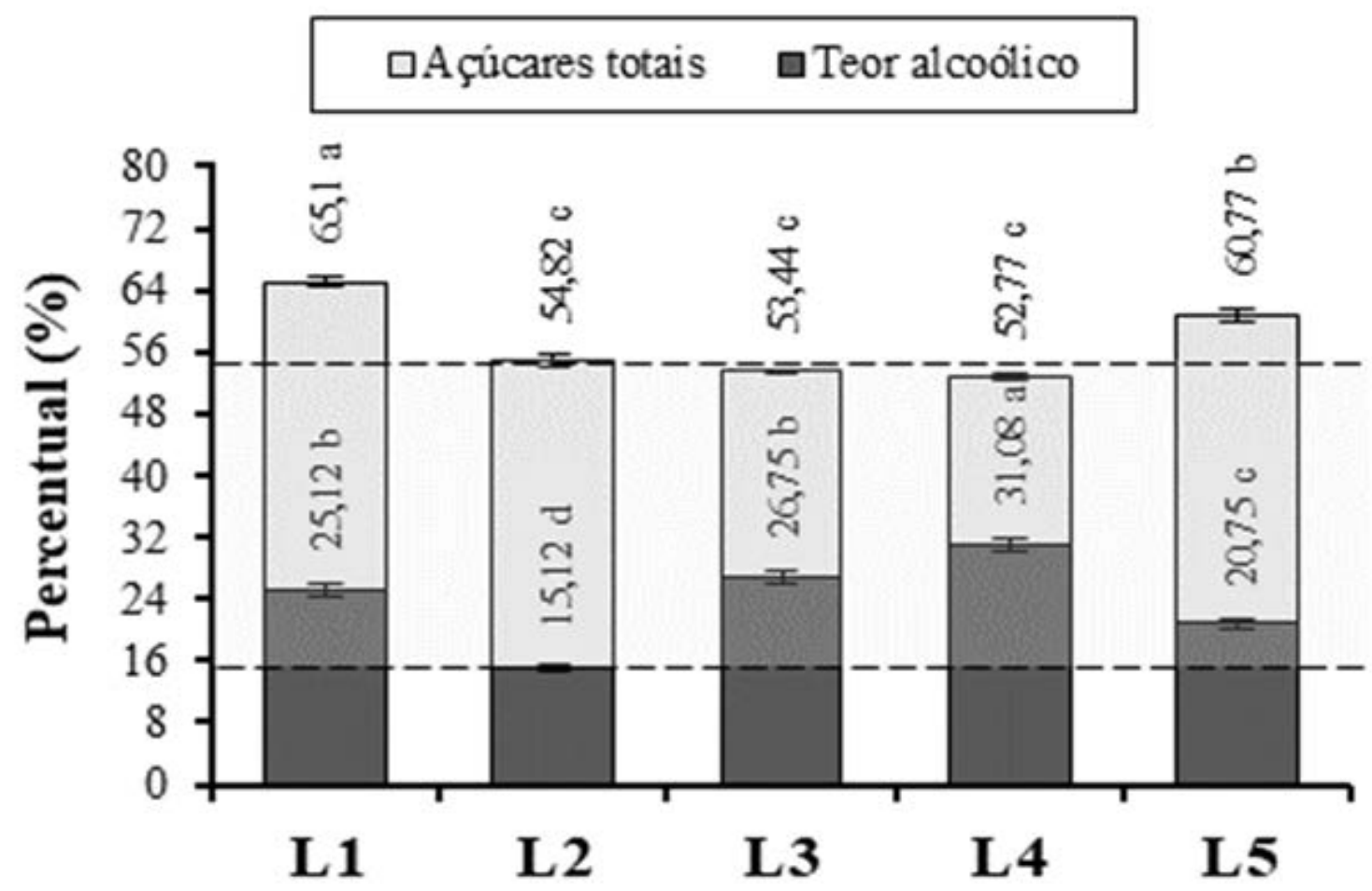

Figura 3. Valores médios de teor alcoólico e açúcares totais dos licores.

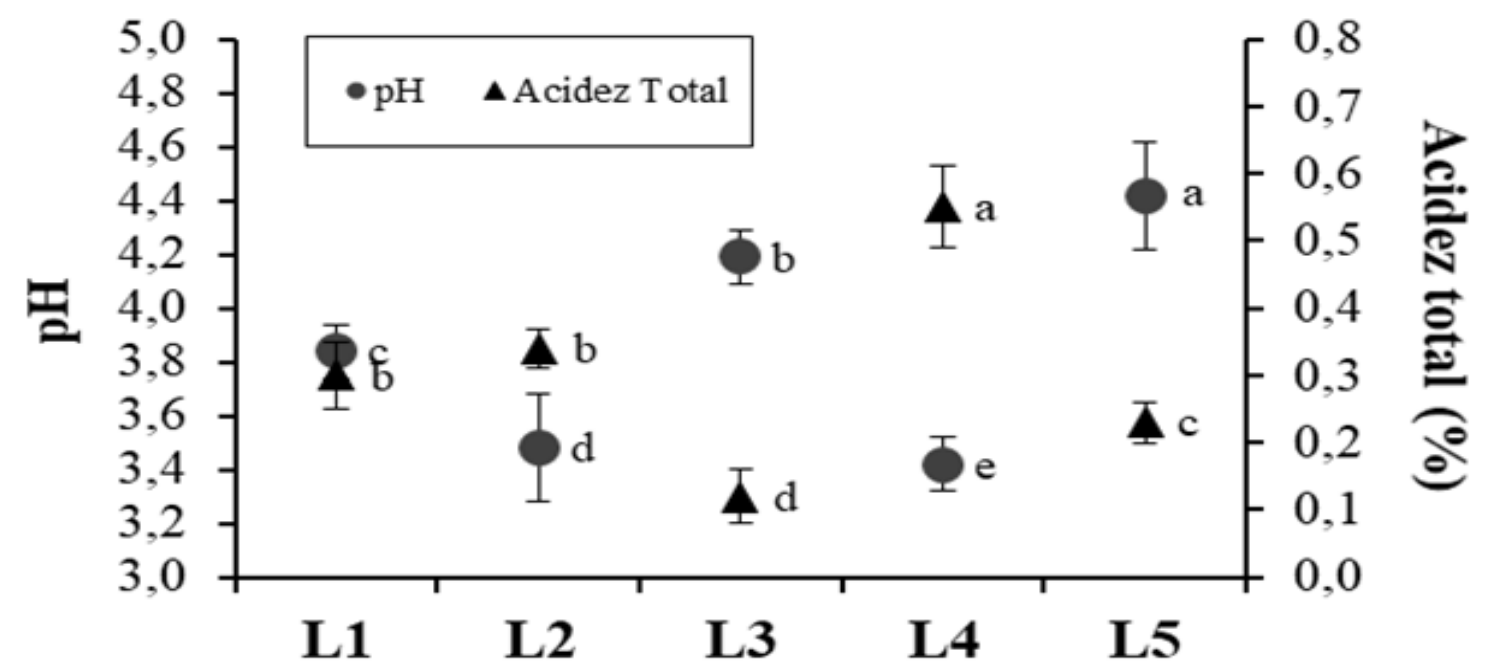

Figura 4. Correlação de pH e acidez total dos licores.

O Decreto no 6.871/2009 (Brasil, 2009), dispõe sobre a padronização, a classificação, o registro, a inspeção, a produção e a fiscalização de bebidas. Para que as bebidas alcoólicas sejam classificadas como licores é exigida graduação alcoólica entre $15 \%$ e 54\% (Brasil, 2008, 2009). Observou-se a conformidade de todos os licores com a legislação vigente quanto a graduação alcoólica, os quais apresentaram diferença significativa entre si $(\mathrm{p}<0,05)$, conforme a Figura 3. 0 licor de graviola apresentou $o$ 
maior teor alcoólico ( $\left.\mathrm{L}_{4}-31,08 \%\right)$ em relação aos demais produtos. 0 licor de acerola apresentou o menor teor alcoólico ( $\left.\mathrm{L}_{2}-15,12 \%\right)$, o que pode estar associado a capacidade de retenção do álcool durante a maceração. Os valores de teor alcoólico obtidos em licor de açaí (Oliveira e Santos, 2011) e licor de corte de morango (Magalhães et al., 2014) foram 16 e $20 \%$, respectivamente.

Pela legislação vigente, os licores elaborados a partir de resíduos agroindustriais nesta pesquisa foram denominados como "tipo creme", contendo na composição mais de $35 \%$ de açúcares totais (Brasil, 2008, 2009). Os licores de abacaxi e manga apresentaram os maiores teores de açúcares totais $\left(L_{1}-65,10 \%\right.$ e $\left.L_{5}-60,70 \%\right)$, possivelmente devido à maior concentração de açúcares nestes resíduos. Os licores de acerola $\left(\mathrm{L}_{2}\right)$, graviola $\left(\mathrm{L}_{4}\right)$ e goiaba $\left(L_{3}\right)$ não diferiram significativamente entre si $(p<0,05)$, com variação dos teores entre 52,77 e $54,82 \%$ de açúcares totais. Valores inferiores foram obtidos por Silva et al. (2017), ao caracterizarem licores de casca de tangerina (28,36\%-34,99\%), sendo denominados de licores finos.

Quanto a correlação de pH e acidez total nos licores tipo creme, observou-se uma faixa de pH entre 3,40 e 4,40, o que indica uma condição limitante para a multiplicação de microrganismos patogênicos e deteriorantes. A baixa acidez total dos produtos pode estar relacionada a concentração reduzida de ácidos orgânicos nos resíduos agroindustriais ou a sua volatilização durante o processamento. Segundo Chitarra e Chitarra (2005), o teor de ácidos nos resíduos de frutas pode ser menor em relação a polpa. Os licores desta pesquisa apresentaram diferença significativa entre si $(\mathrm{p}<0,05)$ para os parâmetros de $\mathrm{pH}$ e acidez total. 0 licor de graviola $\left(\mathrm{L}_{4}\right)$ apresentou maior valor de acidez total e o menor $\mathrm{pH}$, o que pode favorecer o aumento de sua vida de prateleira e uma maior estabilidade dos compostos extraídos pelo processo de maceração alcoólica. Teixeira et al. (2012), ao avaliar licor de abacaxi com diferentes concentrações de açúcar e fruta, obtiveram valores de $\mathrm{pH}$ superiores $(4,40-4,46)$ ao licor de abacaxi $\left(\mathrm{L}_{1}\right)$ da presente pesquisa.

A Tabela 3 expressa os valores médios em base úmida, obtidos na caracterização dos licores tipo creme.

Tabela 3. Valores médios da caracterização dos licores tipo creme em base úmida.

\begin{tabular}{|c|c|c|c|c|c|c|c|}
\hline \multirow{2}{*}{ Parâmetro } & \multicolumn{5}{|c|}{ Licores tipo creme } & \multirow{2}{*}{ MG } & \multirow{2}{*}{$\begin{array}{l}\text { CV } \\
(\%)\end{array}$} \\
\hline & $\mathbf{L}_{1}$ & $\mathbf{L}_{2}$ & $\mathbf{L}_{3}$ & $\mathbf{L}_{4}$ & $\mathbf{L}_{5}$ & & \\
\hline SST ( ${ }^{\circ}$ Brix) & $54,10^{\mathrm{ab}}$ & $50,25^{c}$ & $54,50^{\mathrm{a}}$ & $52,40^{\mathrm{b}}$ & $55,10^{\mathrm{a}}$ & 53,27 & 0,98 \\
\hline Resíduo mineral (\%) & $0,12^{\mathrm{b}}$ & $0,13^{\mathrm{b}}$ & $0,07 \mathrm{c}$ & $0,14^{\mathrm{b}}$ & $0,21^{\mathrm{a}}$ & 0,13 & 7,50 \\
\hline Atividade de água & $0,835^{\mathbf{a}}$ & $0,865^{a}$ & $0,815^{\mathbf{a}}$ & $0,850^{\mathrm{a}}$ & $0,835^{a}$ & 0,840 & 1,99 \\
\hline Antocianinas $\mathrm{mg} / 100 \mathrm{~g}$ & $3,37^{b}$ & $4,86^{\mathrm{a}}$ & - & & $5,38^{a}$ & 4,54 & 5,38 \\
\hline Flavonoides mg/100 g & $0,0054^{\mathrm{d}}$ & $40,72^{\mathrm{a}}$ & $5,75^{c}$ & $7,48^{\mathrm{b}}$ & $0,0342^{\mathrm{d}}$ & 10,79 & 1,28 \\
\hline Luminosidade $\left(\mathrm{L}^{*}\right)$ & $41,01^{b}$ & $8,81^{\mathrm{e}}$ & $42,97 a$ & $36,62^{c}$ & $35,18^{\mathrm{d}}$ & 32,92 & 1,20 \\
\hline dade de vermelho $\left(+a^{*}\right)$ & $7,44^{\mathrm{d}}$ & $24,06^{a}$ & $3,64^{\mathrm{e}}$ & $12,39 \mathrm{~b}$ & $11,22^{c}$ & 11,75 & 0,81 \\
\hline Intensidade de amarelo $\left(+\mathrm{b}^{*}\right)$ & $39,85^{b}$ & $15,00^{\mathrm{d}}$ & $29,08^{c}$ & $44,40^{\mathrm{a}}$ & $44,23^{a}$ & 34,51 & 1,20 \\
\hline Croma $\left(\mathrm{C}^{*}\right)$ & $40,53^{b}$ & $28,35^{c}$ & $29,30^{c}$ & $46,10^{a}$ & $45,62^{\mathrm{a}}$ & 37,98 & 1,10 \\
\hline Ângulo hue $\left(\mathrm{H}^{*}\right)$ & $79,41^{\mathrm{b}}$ & $31,80^{\mathrm{e}}$ & $82,85^{a}$ & $74,37 \mathrm{~d}$ & $75,73^{c}$ & 68,83 & 0,16 \\
\hline
\end{tabular}

$\mathrm{L}_{1}$ - licor de abacaxi, $\mathrm{L}_{2}$ - licor de acerola, $\mathrm{L}_{3}$ - licor de goiaba, $\mathrm{L}_{4}$ - licor de graviola, $\mathrm{L}_{5}$ - licor de manga, STT - sólidos solúveis totais; MG - média geral, CV - coeficiente de variação. Médias seguidas na linha pela mesma letra não diferem significativamente entre si pelo teste de Tukey a $5 \%$ de significância.

Os SST estão relacionados a proporção do xarope adicionado. Não houve diferença significativa $(p<0,05)$ entre os licores de abacaxi $\left(L_{1}\right)$, goiaba $\left(L_{3}\right)$ e manga $\left(L_{5}\right)$. 0 licor de 
manga $\left(\mathrm{L}_{5}\right)$ apresentou 55,10 ${ }^{\circ}$ Brix, que foi superior as demais bebidas, enquanto o licor de acerola $\left(\mathrm{L}_{2}\right)$ apresentou o menor teor, com 50,2 ${ }^{\circ}$ Brix. Resultados inferiores foram encontrados por Magalhães et al. (2014) e Silva et al. (2017), avaliando licor de corte de morango (45 ${ }^{\circ}$ Brix) e licor fino de casca de tangerina (34,00-31,63 ${ }^{\circ}$ Brix), respectivamente. Os elevadores teores dos SST desta pesquisa estão em concordância com a classificação dos licores em "tipo creme".

Quanto ao teor de resíduo mineral, os licores tipo creme de abacaxi $\left(\mathrm{L}_{1}\right)$, acerola $\left(L_{2}\right)$ e graviola $\left(L_{4}\right)$ não apresentaram diferença significativa $(p<0,05)$. Possivelmente, as concentrações de minerais encontradas nos produtos tenham migrado dos resíduos agroindustriais durante o processo de maceração alcoólica. Observou-se nos licores tipo creme uma variação de $0,07 \%$ a $0,21 \%$ de resíduo mineral, o que foi superior ao encontrado por Oliveira e Santos (2011), avaliando licor de açaí (0,05\%), que se baseou em uma polpa e não de um resíduo.

A atividade de água dos licores atingiu uma faixa de variação entre 0,815 e 0,865, sem diferença significativa entre as bebidas $(\mathrm{p}<0,05)$. A baixa atividade de água associada ao baixo $\mathrm{pH}$, teor alcoólico e concentração de sólidos tende a limitar a multiplicação de microrganismos e favorecer a conservação desses produtos.

Observou-se um maior conteúdo de antocianinas nos licores de acerola $\left(\mathrm{L}_{2}-4,86\right.$ $\mathrm{mg} / 100 \mathrm{~g}$ ) e manga ( $\left.\mathrm{L}_{5}-5,38 \mathrm{mg} / 100 \mathrm{~g}\right)$, não ocorrendo diferença significativa entre si $(\mathrm{p}<0,05)$. 0 teor de flavonoides foi superior principalmente no licor de acerola $\left(\mathrm{L}_{2}\right)$, com $40,72 \mathrm{mg} / 100 \mathrm{~g}$, indicando diferença significativa $(\mathrm{p}<0,05)$ entre os licores. 0 licor de abacaxi $\left(L_{1}\right)$ apresentou os menores teores em ambos os compostos, podendo ser justificado pela menor capacidade de retenção de compostos fenólicos durante a maceração ou uma termosensibilidade, durante o processamento e/ou estocagem.

Quanto aos parâmetros de cor, verifica-se que apenas os resultados de intensidade de amarelo $\left(+b^{*}\right)$ e croma $\left(C^{*}\right)$ não apresentaram médias que deferiram significativamente $(p<0,05)$ entre os licores de graviola $\left(L_{4}\right)$ e manga $\left(L_{5}\right)$, assim como os valores de $C^{*}$ para acerola $\left(\mathrm{L}_{2}\right)$ e goiaba $\left(\mathrm{L}_{3}\right)$. Todos os outros resultados para os demais parâmetros apresentaram efeito significativo $(p<0,05)$ entre as médias.

A maioria dos licores apresentaram elevados índices de luminosidade ( $\mathrm{L}^{*}$ ), com valores acima de 35. Apenas o licor de acerola apresentou valor baixo $\left(L_{2}-8,81\right)$ comparado as demais bebidas. Esse baixo índice de $\mathrm{L}^{*}$ do licor de acerola $\left(\mathrm{L}_{2}\right)$ é justificado, visto que o resíduo utilizado era composto por sementes e resíduos de casca, resultando em um produto com coloração muito intensa (Figura 2). Como se pode verificar na Tabela 3, o licor de acerola $\left(\mathrm{L}_{2}\right)$ apresentou-se rico em flavonoides, quando comparado com os demais licores, proporcionando uma coloração mais escura e baixa luminosidade.

0 teor de flavonoides também influenciou em outros parâmetros relacionados a cor das bebidas, garantindo maior destaque também ao licor de acerola $\left(\mathrm{L}_{2}\right)$. Esse licor especificamente revelou a maior intensidade de vermelho $\left(+\mathrm{a}^{*}\right)$, os menores valores de intensidade de amarelo $\left(+b^{*}\right)$ e do ângulo de tonalidade $\left(\mathrm{H}^{*}\right)$.

Os maiores valores de $C^{*}$ foram encontrados nos licores de graviola $\left(L_{4}-46,10\right)$ e manga $\left(L_{5}-45,62\right)$, já os menores foram revelados para os licores de acerola $\left(L_{2}-28,35\right)$ e goiaba $\left(L_{3}-29,30\right)$. Portanto, evidencia-se que a coloração dos licores de graviola $\left(L_{4}\right)$ e manga $\left(L_{5}\right)$ apresentou uma maior saturação de sua cor, ou seja, uma maior pureza.

Os licores tipo creme apresentaram condições higiênico-sanitárias adequadas para o consumo, com baixas contagens para Coliformes a $35{ }^{\circ} \mathrm{C}$ e $45{ }^{\circ} \mathrm{C}(<3 \mathrm{NMP} / \mathrm{g})$, que são indicadores de condições higiênico-sanitárias. De acordo com Nunes et al. (2014), as bebidas alcoólicas atingem a esterilidade comercial com aproximadamente $16^{\circ} \mathrm{GL}$.

$\mathrm{Na}$ Tabela 4 estão apresentados os valores médios da aceitação sensorial e intenção de compra dos licores tipo creme. Os Índices de Aceitabilidade foram expressos na Figura 5, visando à avaliação do potencial mercadológico individual das bebidas para comercialização. 
Tabela 4. Aceitação sensorial e intenção de compra dos licores tipo creme.

\begin{tabular}{lccccccc}
\hline \multirow{2}{*}{\multicolumn{1}{c}{ Atributos }} & \multicolumn{9}{c}{ Licores tipo creme } & \multirow{2}{*}{ MG } & \multirow{2}{*}{ CV (\%) } \\
\cline { 2 - 6 } & $\mathrm{L}_{1}$ & $\mathrm{~L}_{2}$ & $\mathrm{~L}_{3}$ & $\mathrm{~L}_{4}$ & $\mathrm{~L}_{5}$ & & \\
\hline Cor & $7,14^{\mathrm{a}}$ & $6,81^{\mathrm{ab}}$ & $7,09^{\mathrm{a}}$ & $7,51^{\mathrm{a}}$ & $6,09^{\mathrm{b}}$ & 6,92 & 24,39 \\
Aparência & $7,30^{\mathrm{a}}$ & $6,75^{\mathrm{ab}}$ & $7,12^{\mathrm{a}}$ & $7,25^{\mathrm{a}}$ & $6,25^{\mathrm{b}}$ & 6,93 & 22,96 \\
Aroma & $6,93^{\mathrm{a}}$ & $6,42^{\mathrm{ab}}$ & $6,88^{\mathrm{a}}$ & $6,72^{\mathrm{ab}}$ & $5,75^{\mathrm{b}}$ & 6,54 & 28,89 \\
Consistência & $7,18^{\mathrm{a}}$ & $7,19^{\mathrm{a}}$ & $6,91^{\mathrm{ab}}$ & $7,49^{\mathrm{a}}$ & $6,23^{\mathrm{b}}$ & 7,00 & 22,68 \\
Sabor & $7,00^{\mathrm{a}}$ & $6,42^{\mathrm{ab}}$ & $6,39^{\mathrm{ab}}$ & $6,75^{\mathrm{a}}$ & $5,32^{\mathrm{b}}$ & 6,37 & 35,38 \\
Doçura & $7,25^{\mathrm{a}}$ & $6,86^{\mathrm{a}}$ & $6,81^{\mathrm{a}}$ & $6,91^{\mathrm{a}}$ & $5,58^{\mathrm{b}}$ & 6,67 & 27,69 \\
PTA & $7,18^{\mathrm{a}}$ & $6,32^{\mathrm{ab}}$ & $6,65^{\mathrm{ab}}$ & $6,81^{\mathrm{a}}$ & $5,79^{\mathrm{b}}$ & 6,54 & 28,63 \\
Impressão global & $7,14^{\mathrm{a}}$ & $6,65^{\mathrm{ab}}$ & $6,63 \mathrm{ab}$ & $7,00^{\mathrm{a}}$ & $5,88^{\mathrm{b}}$ & 6,65 & 26,97 \\
\hline Intenção de compra & $3,75^{\mathrm{a}}$ & $3,42^{\mathrm{ab}}$ & $3,58^{\mathrm{a}}$ & $3,79^{\mathrm{a}}$ & $2,86^{\mathrm{b}}$ & 3,48 & 34,37 \\
\hline
\end{tabular}

$\mathrm{L}_{1}$ - licor de abacaxi, $\mathrm{L}_{2}$ - licor de acerola, $\mathrm{L}_{3}$ - licor de goiaba, $\mathrm{L}_{4}$ - licor de graviola, $\mathrm{L}_{5}$ - licor de manga; PTA - percepção do teor alcoólico; MG - média geral, DMS - desvio médio significativo. Médias seguidas na linha pela mesma letra não diferem significativamente entre si pelo teste de Tukey a 5\% de significância.

Os licores tipo creme elaborados com resíduos da indústria de polpas de frutas apresentaram pelos provadores boa aceitação sensorial na maioria dos atributos. A bebida alcoólica de manga $\left(\mathrm{L}_{5}\right)$ indicou numericamente as menores notas em média, com Índice de Aceitabilidade (IA) menor que 70\%.

Observou-se diferenças significativas $(\mathrm{p}<0,05)$ entre pelo menos dois dos produtos para cada atributo avaliado (Tabela 4). Quanto a cor e aparência, a maioria dos licores tipo creme desenvolveram durante o processo de maceração uma coloração própria, contribuindo para a aceitação que atingiu o termo hedônico "gostei moderadamente".

Para um produto ser cogitado como potencial para testes mercadológicas e sua comercialização, Gularte (2009) afirma que o IA deve estar $\geq 70 \%$. Assim, com exceção do licor de manga $\left(\mathrm{L}_{5}\right)$, todos as bebidas ultrapassaram $70 \%$, chegando o licor de graviola $\left(\mathrm{L}_{4}\right)$ a $83,44 \%$ no atributo cor.

Os atributos aroma, consistência, sabor e doçura estão mais caracterizados pelo tipo de processamento dos licores, através do qual migraram compostos e as propriedades foram definidas. Com destaque para os atributos citados, as médias das notas do licor tipo creme de abacaxi $\left(L_{1}\right)$ se sobressaíram com melhor aceitação em relação as demais bebidas.

O aroma e sabor foram marcados pelas características próprias dos resíduos agroindustriais das frutas, bem como a doçura foi intensificada pela adição do xarope. Os termos hedônicos variaram entre "gostei ligeiramente" e "gostei moderadamente", sendo que no IA as médias superaram 70\%, atingindo $83,22 \%$ no atributo consistência do licor de graviola $\left(\mathrm{L}_{4}\right)$.

Quanto a percepção do teor alcoólico, percebe-se um contraste com os resultados do teor alcoólico (Figura 3). 0 licor tipo creme de abacaxi $\left(\mathrm{L}_{1}\right)$ foi o mais bem aceito, com média de 7,18, que representa o termo hedônico "gostei moderadamente". Do mesmo modo na impressão global, o licor de manga $\left(L_{5}\right)$ apresentou a menor aceitação sensorial, com IA de 65,33\%.

Esse fato pode ser justificado pela necessidade de mais testes nas formulações, visando uma melhor aceitação em detrimento da concentração de resíduos agroindustriais e xarope. Logo, em relação a intenção de compra, observou-se que as notas atingiram os termos "possivelmente compraria o produto" e "talvez comprasse, talvez não comprasse", com exceção do licor tipo creme de manga $\left(L_{5}\right)$. 

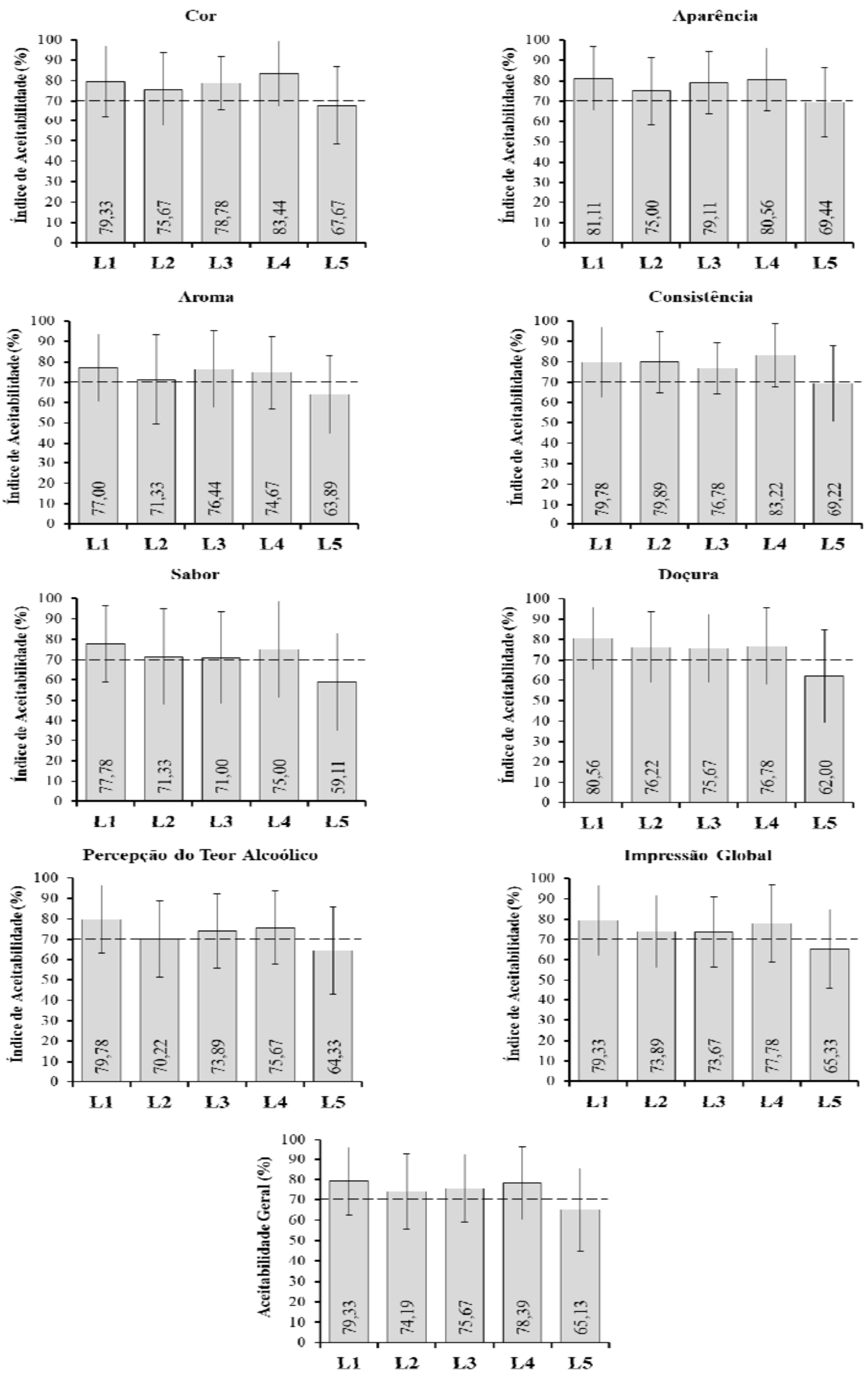

Figura 5. Índices de Aceitabilidade e aceitação geral dos licores tipo creme. $\mathrm{L}_{1}$ - licor de abacaxi, $\mathrm{L}_{2}$ licor de acerola, $\mathrm{L}_{3}$ - licor de goiaba, $\mathrm{L}_{4}$ - licor de graviola, $\mathrm{L}_{5}$ - licor de manga. 
Verificou-se ser viável aplicar testes mercadológicos para comercialização em detrimento dos elevados IA, intenção e compra e aceitabilidade geral. A aceitabilidade geral alcançou importantes percentuais de até 79,33\% para o licor tipo creme de abacaxi $\left(\mathrm{L}_{1}\right)$ e $78,39 \%$ para o licor tipo creme de graviola $\left(\mathrm{L}_{4}\right)$.

Na Tabela 5, encontra-se a cinética de degradação colorimétrica dos licores tipo creme. Observa-se que os modelos cinéticos de zero, primeira e segunda ordem ajustaramse com variação aos dados experimentais dos parâmetros luminosidade ( $\mathrm{L}^{*}$ ), intensidade de vermelho $\left(+a^{*}\right)$ e de intensidade de amarelo $\left(+b^{*}\right)$ obtidos na cinética de degradação.

Tabela 5. Análises de cor durante a cinética de degradação colorimétrica dos licores tipo creme, conforme os modelos cinéticos de zero, primeira e segunda ordem e os tempos de meia vida.

\begin{tabular}{|c|c|c|c|c|c|c|c|c|c|c|}
\hline \multirow{2}{*}{$\begin{array}{l}\text { Licore } \\
\text { s tipo } \\
\text { creme }\end{array}$} & \multirow[b]{2}{*}{ Modelos } & \multicolumn{3}{|c|}{ Luminosidade } & \multicolumn{3}{|c|}{ Intensidade de vermelho } & \multicolumn{3}{|c|}{ Intensidade de amarelo } \\
\hline & & $k\left(\right.$ dia $\left.^{-1}\right)$ & $\mathrm{R}^{2}$ & $\theta^{1 / 2}$ (dia) & $k\left(\right.$ dia $\left.^{-1}\right)$ & $\mathrm{R}^{2}$ & $\begin{array}{c}\theta^{1 / 2} \\
\text { (dia) }\end{array}$ & $k\left(\right.$ dia $\left.^{-1}\right)$ & $\mathrm{R}^{2}$ & $\begin{array}{c}\theta^{1 / 2} / 2 \\
\text { (dia) }\end{array}$ \\
\hline \multirow{3}{*}{$\mathrm{L}_{1}$} & $\begin{array}{c}\text { Zero } \\
\text { ordem }\end{array}$ & 0,519456 & 0,9690 & 39,44 & 0,416123 & 0,9808 & 9,05 & 0,956314 & 0,9607 & 21,29 \\
\hline & $\begin{array}{c}\text { Primeira } \\
\text { ordem }\end{array}$ & 0,013793 & 0,9631 & - & 0,093399 & 0.9070 & - & 0,025654 & 0,8992 & - \\
\hline & $\begin{array}{c}\text { Segunda } \\
\text { ordem }\end{array}$ & 0,000379 & 0,9688 & - & 0,034521 & 0,7963 & - & 0,000918 & 0,8901 & - \\
\hline \multirow{3}{*}{$\mathrm{L}_{2}$} & $\begin{array}{c}\text { Zero } \\
\text { ordem }\end{array}$ & 0,459064 & 0,9849 & 9,36 & 1,021270 & 0,9236 & 12,9 & 0,795647 & 0,9858 & 9,22 \\
\hline & $\begin{array}{c}\text { Primeira } \\
\text { ordem }\end{array}$ & 0,087514 & 0,9620 & - & 0,044758 & 0,7882 & - & 0,089578 & 0,9587 & - \\
\hline & $\begin{array}{c}\text { Segunda } \\
\text { ordem }\end{array}$ & 0,022740 & 0,9121 & - & 0,003729 & 0,8282 & - & 0,014062 & 0,9082 & - \\
\hline \multirow{3}{*}{$\mathrm{L}_{3}$} & $\begin{array}{c}\text { Zero } \\
\text { ordem }\end{array}$ & 0,249774 & 0,9540 & 85,59 & 0,175373 & 0,9526 & 11,11 & 0,579873 & 0,9598 & 25,83 \\
\hline & $\begin{array}{c}\text { Primeira } \\
\text { ordem }\end{array}$ & 0,006551 & 0,9338 & - & 0,056847 & 0,7948 & - & 0,018702 & 0,8869 & - \\
\hline & $\begin{array}{c}\text { Segunda } \\
\text { ordem }\end{array}$ & 0,000147 & 0,9595 & - & 0,033174 & 0,7541 & - & 0,000898 & 0,9215 & - \\
\hline \multirow{3}{*}{$\mathrm{L}_{4}$} & $\begin{array}{l}\text { Zero } \\
\text { ordem }\end{array}$ & 0,376480 & 0,9741 & 48,79 & 0,334564 & 0,9558 & 19,18 & 0,517897 & 0,9710 & 42,93 \\
\hline & $\begin{array}{c}\text { Primeira } \\
\text { ordem }\end{array}$ & 0,010614 & 0,9634 & - & 0,028147 & 0,9286 & - & 0,018702 & 0,9625 & - \\
\hline & $\begin{array}{c}\text { Segunda } \\
\text { ordem }\end{array}$ & 0,000328 & 0,9727 & - & 0,003219 & 0,9627 & - & 0,000317 & 0,9672 & - \\
\hline \multirow{3}{*}{$\mathrm{L}_{5}$} & $\begin{array}{c}\text { Zero } \\
\text { ordem }\end{array}$ & 0,591750 & 0,9748 & 30,13 & 0,457300 & 0,9703 & 12,58 & 0,929103 & 0,6348 & 25,38 \\
\hline & $\begin{array}{l}\text { Primeira } \\
\text { ordem }\end{array}$ & 0,017125 & 0,9606 & - & 0,053190 & 0,8890 & - & 0,017371 & 0,4807 & - \\
\hline & $\begin{array}{c}\text { Segunda } \\
\text { ordem }\end{array}$ & 0,000609 & 0,9796 & - & 0,008786 & 0,8596 & - & 0,000733 & 0,5343 & - \\
\hline
\end{tabular}

$\mathrm{L}_{1}$ - licor de abacaxi, $\mathrm{L}_{2}$ - licor de acerola, $\mathrm{L}_{3}$ - licor de goiaba, $\mathrm{L}_{4}$ - licor de graviola, $\mathrm{L}_{5}$ - licor de manga.

De modo geral, o melhor ajuste para a colorimetria foi o modelo de ordem zero por apresentar os maiores valores de $\mathrm{R}^{2}>0,91$, exceto para a intensidade de amarelo $\left(+\mathrm{b}^{*}\right)$, que apresentou um ajuste de 0,63. A maioria das reações estudadas em alimentos são caracterizadas como cinéticas de ordem zero ou primeira ordem (Taoukis e Labuza, 1996). 
Comportamento semelhante foi observado para a alteração da cor do azeite de pequi, que seguiu a cinética de ordem zero (Rodrigues et al., 2013). Porém, vários estudos relataram que a degradação da cor em temperaturas até $100{ }^{\circ} \mathrm{C}$ durante o processamento de alimentos ou durante o armazenamento segue um modelo cinético de primeira ordem: xarope de morango (Skrede et al., 1992), suco concentrado de cenoura preta (Türkyilmaz e Özkan, 2012); com valores dos coeficientes de determinação variando de 0,68 a 0,91.

A constante da velocidade da reação $(k)$ é um indicador que permite a predição da degradação térmica dos parâmetros estudados e quanto menor o valor de $k$, melhor a estabilidade (Loypimai et al., 2016). Seguindo o modelo de zero ordem, já que apresentou os melhores ajustes, porém também se verifica para os modelos de primeira e segunda ordem que o licor de goiaba $\left(\mathrm{L}_{3}\right)$ apresentou melhor estabilidade quanto a luminosidade $\left(L^{*}\right)$; e o de menor estabilidade foi o licor de manga $\left(L_{5}\right)$, indicando que a velocidade da reação aumentou.

Em relação à intensidade de vermelho e amarelo verifica-se que o licor de goiaba $\left(\mathrm{L}_{3}\right)$ apresentou a menor velocidade de reação $(k)$ para a intensidade de vermelho, e para a intensidade de amarelo foi o licor de graviola $\left(\mathrm{L}_{4}\right)$. Comportamento inverso, ou seja, de menor estabilidade foi verificado para o licor de acerola $\left(L_{2}\right)$ para a intensidade de vermelho, e licor de abacaxi $\left(\mathrm{L}_{1}\right)$ para a intensidade de amarelo.

Zozio et al. (2011) verificaram que os valores de $k$ aumentaram para as antocianinas em bebidas de extratos de amora preta, açaí e cenoura preta, armazenadas nas temperaturas de 20,30 e $50{ }^{\circ} \mathrm{C}$. As diferenças nos valores de $k$ podem estão relacionadas à composição física e química do produto em que as antocianinas estão presentes, tendo, por exemplo, influência dos compostos fenólicos, íons e pH (Sinela et al., 2017).

Constata-se que o tempo de meia vida ${ }^{\left(\boldsymbol{\theta}_{1 / 2}\right)}$ dos licores apresentaram variação para os parâmetros estudados, o licor de goiaba $\left(\mathrm{L}_{3}\right)$ apresentou o maior tempo de meia vida para a luminosidade, licor de graviola $\left(\mathrm{L}_{4}\right)$ para a intensidade de vermelho e amarelo. Menor vida para a luminosidade foi o licor de acerola $\left(\mathrm{L}_{2}\right)$, intensidade de vermelho o licor de abacaxi $\left(\mathrm{L}_{1}\right)$ e intensidade de amarelo o licor de acerola $\left(\mathrm{L}_{2}\right)$.

A degradação da cor é causada principalmente por oxidação, clivagem da ligação covalente ou reações avançadas de oxidação devido ao processamento térmico (Patras et al., 2010).

\section{Conclusões}

Os licores tipo creme elaborados com resíduos agroindustriais da indústria de polpa de frutas atenderam aos padrões de identidade e qualidade preconizados pela legislação, com condições higiênico-sanitárias adequadas para o consumo.

Os parâmetros físicos e químicos indicaram baixa atividade de água associada ao baixo $\mathrm{pH}$, teor alcoólico e concentração de sólidos, que podem favorecer a conservação das bebidas. 0 licor de goiaba foi o mais estável durante a cinética de degradação da cor para luminosidade, enquanto o licor de manga apresentou menos estabilidade.

O licores apresentaram boa aceitação sensorial, principalmente o licor tipo creme de abacaxi e graviola, com índice de aceitabilidade acima de 70\%. Portanto, o processamento de licores com resíduos agroindustriais é viável e pode ser mais uma fonte de renda para o pequeno produtor de polpa de frutas.

\section{Conflito de interesses}

Os autores declaram não haver conflito de interesses. 


\section{Referências}

ABRAFRUTAS - Associação Brasileira dos Produtores Exportadores de Frutas e Derivados. Estatística de exportações de frutas em 2019. 2019. Disponível em: <https://abrafrutas.org/2020/01/28/8825/>. Acesso em: 16 abr. 2020.

Brasil. Decreto no 6.871, de 4 de junho de 2009. Regulamenta a Lei no 8.918, de 14 de julho de 1994. Dispõe sobre a padronização, a classificação, o registro, a inspeção, a produção e a fiscalização de bebidas. Disponível em: <http://www.planalto.gov.br/ ccivil_03/_Ato2007-2010/2009/Decreto/D6871.htm>. Acesso em: 16 abr. 2020.

Brasil. Instrução Normativa MAPA no 55, de 31 de outubro de 2008. Aprova os regulamentos técnicos para a fixação dos padrões de identidade e qualidade para as bebidas alcoólicas por mistura: licor, bebida alcoólica mista, batida, caipirinha, bebida alcoólica composta, aperitivo e aguardente composta. Disponível em: <http://www.agricultura.gov.br/assuntos/vigilancia-agropecuaria/ivegetal/bebidasarquivos/in-no-55-de-18-de-novembro-de-2009.doc>. Acesso em: 16 abr. 2020.

Chitarra, M. I. F.; Chitarra, A. B. Pós-colheita de frutos e hortaliças: fisiologia e manuseio. 2. ed. Lavras: UFLA, 2005.

Coelho, E. M.; Viana, A. C.; Azevedo, L. C. Prospecção tecnológica para o aproveitamento de resíduos industriais, com foco na indústria de processamento de manga. Cadernos de Prospecção, v. 7, n. 4, p. 550-560, 2014. http://doi.org/10.9771/s.cprosp.2014.007.056

Dutcosky, S. D. Análise sensorial de alimentos. 4. ed. Curitiba: Champagnat, 2013.

Feitosa, B. F.; Oliveira, E. N. A.; Oliveira Neto, J. O.; Oliveira, D. B.; Feitosa, R. M. Cinética de secagem dos resíduos da agroindústria processadora de polpas de frutas. Energia na Agricultura, v. 34, n. 1, p. 134-141, 2019. https://doi.org/10.17224/EnergAgric.2019 v34n01p134-141

Francis, F. J. Analysis of anthocyanins. In: Markakis, P. Anthocyanins as food colors. London: Academic Press, 1982. p. 181-206.

Gularte, M. A. Análise sensorial. Pelotas: Editora Universitária da Universidade Federal de Pelotas, 2009.

IAL - Instituto Adolfo Lutz. Métodos físico-químicos para análise de alimentos. 4. ed. São Paulo: IAL, 2008.

Infante, J.; Selani, M. M.; Toledo, M. N. V.; Silveira-Diniz, M. F.; Alencar, S. M.; Spoto, M. H. F. Atividade antioxidante de resíduos agroindustriais de frutas tropicais. Alimentos e Nutrição, v. 24, n. 1, p. 87-91, 2013.

Loypimai, P.; Moongngarm, A.; Chottanom, P. Thermal and pH degradation kinetics of anthocyanins in natural food colorant prepared from black rice bran. Journal of Food Science and Technology, v. 53, n. 1, p. 461-470, 2016. https://doi.org/10.1007/s13197015-2002-1

Magalhães, D. C.; Andrade, R. O.; Costa, D. P.; Santos, D. B.; Cardoso, R. L. Desenvolvimento, caracterização físico-química e sensorial de licor de corte de morango. Enciclopédia Biosfera, v. 10, n. 18, p. 1193, 2014.

Nunes, S. P.; Teixeira, L. J. Q.; Silva, G. B.; Bernardes, P. C.; Rodrigues Júnior, S. Influência do teor alcoólico na sobrevivência de Staphylococcus aureus em simulados de bebidas alcoólicas. Revista Brasileira de Produtos Agroindustriais, v. 16, n. 3, p. 255-264, 2014. 
Oliveira, E. N. A.; Feitosa, B. F.; Souza, R. L A. Tecnologia e processamento de frutas: doces, geleias e compotas. 1. ed. Natal: Editora IFRN, 2018.

Oliveira, E. N. A; Santos, D. C. Processamento e avaliação da qualidade de licor de açaí (Euterpe oleracea Mart.). Revista do Instituto Adolfo Lutz, v. 70, n. 4, p.534-541, 2011.

Oliveira, E. N. A.; Santos, D. C.; Santos, Y. M. G.; Oliveira, F. A. A. Aproveitamento agroindustrial da graviola (Annona muricata L.) para produção de licores: avaliação sensorial. Journal of Biotechnology and Biodiversity, v. 7, n. 2, p. 281-290, 2019. https://doi.org/10.20873/jbb.uft.cemaf.v7n2.alvesoliveira

Patras, A.; Brunton, N. P.; O'donnell, C.; Tiwarib, B. K. Effect of thermal processing on anthocyanin stability in foods; mechanisms and kinetics of degradation. Trends in Food $\begin{array}{lllllll}\text { Science } \& \text { Technology, } & \text { v. 21, } & \text { n. 1, } & \text { p. 3-11, 2010. https://doi.org/10.1016/ }\end{array}$ j.tifs.2009.07.004

Rodrigues, M. L.; Souza, A. R. M.; Lima, J. C. R.; Moura, C. J.; Geraldine, R. M. Cinética da degradação de carotenoides e da alteração de cor do azeite de pequi submetido ao aquecimento em temperatura de fritura. Ciência Rural, v. 43, n. 8, p. 1509-1515, 2013. https://doi.org/10.1590/S0103-84782013000800027

Santos, K. M.; Machado, M. A.; Gomes, P. O. M. Caracterização físico-química, determinação de minerais e avaliação do potencial antioxidante de licores produzidos artesanalmente. Multi-Science Journal, v. 1, n. 12, p. 54-61, 2018. https://doi.org/10.33837/ msj.v1i12.583

SEBRAE - Serviço Brasileiro de Apoio às Micro e Pequenas Empresas. Segmento de licores se reinventa e surpreende. 2014. Disponível em: <https://respostas.sebrae.com.br/ segmento-de-licores-se-reinventa-e-surpreende/>. Acesso em: 16 abr. 2020.

Sensing, K. M. Comunicação precisa da cor. Sakai-Osaka, Japão: AEBDPK, 1998.

Silva, F. A. Z.; Azevedo, C. A. V. The assistat software version 7.7 and its use in the analysis of experimental data. African Journal of Agricultural Research, v. 11, n. 3, p. 3733-3740, 2016. https://doi.org/10.5897/AJAR2016.11522

Silva, N.; Junqueira, V. C. A.; Silveira, N. F. A.; Taniwaky, N. H.; Gomes, R. A. R.; Okazari, M. M. Manual de métodos de análise microbiológica de alimentos e água. 5. ed. São Paulo: Blücher, 2017.

Silva, R. C.; Amorim, A. B. F.; Feitosa, R. M.; Oliveira, E. N. A.; Feitosa, B. F.; Amadeu, L. T. S. Licor fino de casaca de tangerina: processamento e caracterização. Arquivos Brasileiros de Alimentação, v. 2, n. 3, p. 164-173, 2017.

Sinela, A.; Rawat, N.; Mertz, C.; Achir, N.; Fulcrand, H.; Dornier, M. Anthocyanins degradation during storage of Hibiscus sabdariffa extract and evolution of its degradation products. Food Chemistry, v. 214, n. 1, p. 234-241, 2017. https://doi.org/10.1016/ j.foodchem.2016.07.071

Skrede, G.; Wrolstad, R.E.; Lea, P.; Enersen, G. Color stability of strawberry and blackcurrant syrups. Journal of Food Science, v. 57, n. 1, p. 172-177, 1992. https://doi.org/10.1111/j.1365-2621.1992.tb05449.x

Taoukis, P.; Labuza, T. P. Summary: Integrative concepts (shelf-life testing and modelling). In: Fennema, O. (Ed.). Food chemistry. 3. ed. New York: Marcel Dekker, 1996. p. 1013-1042. 
Teixeira, L. J. Q.; Simões, L. S.; Saraiva, S. H.; Junqueira, M. S.; Sarton, M. A. Determinação da proporção de açúcares e fruta necessários para conferir os atributos ideias ao licor de abacaxi. Enciclopédia Biosfera, v. 8, p. 1883-1889, 2012.

Türkyilmaz, M.; Özkan, M. Kinetics of anthocyanin degradation and polymeric colour formation in black carrot juice concentrates during storage. International Journal of Food Science \& Technology, v. 47, n. 11, p. 2273-2281, 2012. https://doi.org/10.1111/ j.1365-2621.2012.03098.x

Uchôa Thomaz, A. M. A.; Sousa, E. C.; Lima, A.; Lima, R. M. T.; Freitas, P. A. P.; Souza, M. A. M.; Thomaz, J. C. A.; Carioca, J. O. B. Elaboração e aceitabilidade de produtos de panificação enriquecidos com sementes de goiaba (Psidium guajava) em pó. HOLOS, v. 5, 2014. https://doi.org/10.15628/holos.2014.1895

Zhishen, J.; Mengcheng, T.; Jianming, W. The determination of flavonoid contents in mulberry and their scavenging effects on superoxide radicals. Food Chemistry, v. 64, n. 4, p. 555-559, 1999. https://doi.org/10.1016/S0308-8146(98)00102-2

Zozio, S.; Pallet, D.; Dornier, M. Evaluation of anthocyanin stability during storage of a coloured drink made from extracts of the Andean blackberry (Rubus glaucus Benth.), açai (Euterpe oleracea Mart.) and black carrot (Daucus carota L.). Fruits, v. 66, n. 3, p. 203-215, 2011. https://doi.org/10.1051/fruits/2011030

Informação da Licença: Este é um artigo Open Access distribuído sob os termos da Licença Creative Commons Attribution, que permite uso irrestrito, distribuição e reprodução em qualquer meio, desde que a obra original seja devidamente citada. 